\title{
BMP Signaling Regulates Extracellular Matrix Composition and Permeability in C.elegans
}

\author{
R.D. Schultz ${ }^{1}$, E.A. Ellis ${ }^{2}$, and T.L. Gumienny ${ }^{1}$ \\ ${ }^{1}$ Molecular and Cellular Medicine, Texas A\&M University Health Science Center, College Station, \\ TX, USA. \\ ${ }^{2}$ Microscopy and Imaging Center, Texas A\&M University, College Station, TX, USA.
}

Bone morphogenetic protein (BMP) pathway signaling plays a critical role in extracellular growth and homeostasis, including basement membrane remodeling and long bone growth. In the small free-living nematode $C$. elegans, BMP signaling regulates body size development and drug response through an unknown physiological mechanism. Our lab and others have shown animals lacking BMP signaling $(b m p(-))$ are short and display increased sensitivity to anesthetics, while animals overproducing BMP $((b m p(++))$ are long and display resistance to anesthetics [1]. Signaling factors or organization of the cuticle, an extracellular matrix that surrounds and protects the animal from its environment, can influence body shape [2]. Further, large-scale gene expression studies have shown that some cuticular collagen are highly regulated by BMP signaling [3-5]. Based on these data, we tested the hypothesis that BMP affects body length and drug response through organization of the nematode cuticle.

Using a novel, microwave-based technique in live nematodes, we tested cuticular permeability barrier function by staining animals with the cuticle-impermeable nuclear dye, Hoechst 33342 in the PELCO BioWave ${ }^{\circledR}$ microwave. We found that animals deficient in BMP signaling display increased uptake of the nuclear stain, compared to animals with either wild-type or increased BMP pathway signaling (Fig. 1). These results indicate that BMP affects cuticular permeability, causing increased dye uptake and drug sensitivity in animals with decreased BMP signaling. Through a serendipitous discovery, we found that $b m p(-)$ populations suspended in buffer formed clumps, by individuals adhering to each other by their tail tips (Fig. 2). These 'worm-stars' were never observed in wild-type or bmp(++) animals. The outermost layer on the cuticle is a negatively charged surface coat. We found that by stripping the cuticle's surface coat with ethanol causes wild-type animals to also clump in liquid, suggesting that ionic properties of the cuticle contribute to worm-star formation. These results further support a role for BMP signaling in regulating surface cuticular properties.

We examined the cuticle directly using TEM. As the cuticle of $C$. elegans is extremely tough, we optimized methods for sample preparation using the microwave, eliminating any need for cuticle cutting during specimen preparation. To preserve and stain lipids that are normally stripped during dehydration, we included malachite green in the primary fixative [6]. The cuticle is composed of multiple layers that differ in both composition and organization and are clearly visible under longitudinal ridges called alae (Fig. 3). We found that BMP levels affect alar width, but not alar depth (Table 1). Malachite green stains the lipids on the outermost layer of the cuticle, which we observed to be severely depleted in animals lacking BMP signaling, while animals with increased BMP signaling display an increased concentration of lipids relative to wild type (Fig. 3). We also found BMP dose affects the medial layer, where $b m p(-)$ animals displayed a strikingly dense medial layer compared to wild-type or $b m p(++)$ animals (Fig. 3). BMP levels also affect the depth of the medial layer, where decreased or increased BMP signaling is associated with a decrease in medial layer depth (Table 1). Finally, we found that the top-most basal layer of oriented collagens is less organized in some $b m p(++)$ animals (Fig. 3). These results reveal a 
role for BMP signaling in organization and composition of the cuticle and provide a model for the physiological mechanism for alteration of both body size and drug response in BMP variant animals.

\section{References}

[1] RD Schultz et al, PLoS One, Under Revision. [2] L McMahon et al, Mol Biol Cell 14 (2003), p. 1366. [3] M Mochii et al, Proc Natl Acad Sci USA 96 (1999), p. 15020. [4] J Liang et al, Dev Biol 305 (2007), p. 714. [5] AF Roberts et al, BMC Developmental Biology 10 (2010), p. 61. [6] RJ Teichman et al, Biology of Reproduction 7 (1972), p. 73.

[7] This work was supported by NIH 1R01GM097591-01 and by TAMHSC MCMD start-up funds.
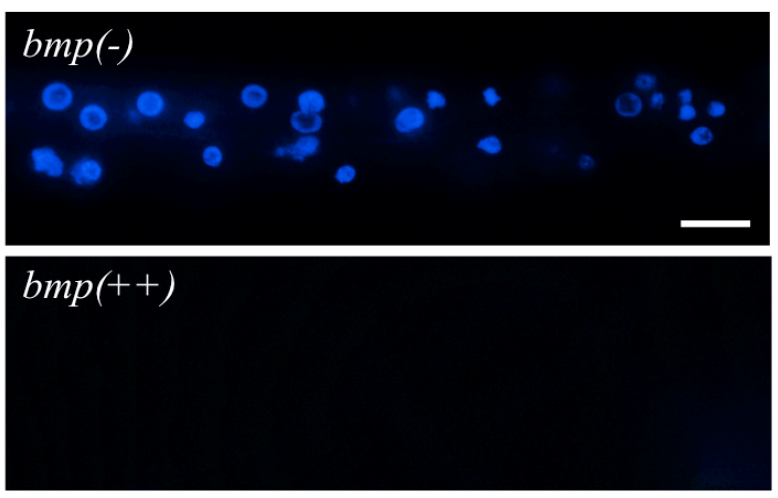

Fig. 1. Hoechst 33342 staining in animals with decreased (top) or increased BMP signaling (bottom) reveals differences in cuticular permeability. Scale bar $=10 \mu \mathrm{m}$.

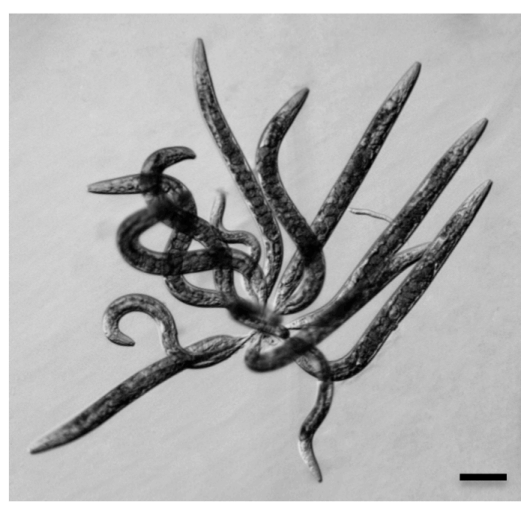

Fig. 2. Loss of BMP signaling causes animals to form worm-stars when suspended in liquid. Scale bar $=100 \mu \mathrm{m}$.

\begin{tabular}{|llll|}
\hline Genotype & Alar width $(\mu \mathrm{m})$ & Alar depth $(\mu \mathrm{m})$ & Medial layer depth $(\mu \mathrm{m})$ \\
bmp(-) & $2.51 \pm 0.34^{*}$ & $0.98 \pm 0.09$ & $0.22 \pm 0.02^{*}$ \\
Wild type & $3.06 \pm 0.15$ & $1.15 \pm 0.16$ & $0.40 \pm 0.09$ \\
bmp(++) & $2.42 \pm 0.23^{* *}$ & $0.89 \pm 0.14$ & $0.24 \pm 0.08^{*}$ \\
\hline
\end{tabular}

Table 1. Comparison of cuticular components in animals with varying levels of BMP signaling. Values indicate the mean $\pm \mathrm{SD}$. P-values (t-test) compare data to wild type $(* * \mathrm{P} \leq 0.001 ; * \mathrm{P} \leq 0.01)$.

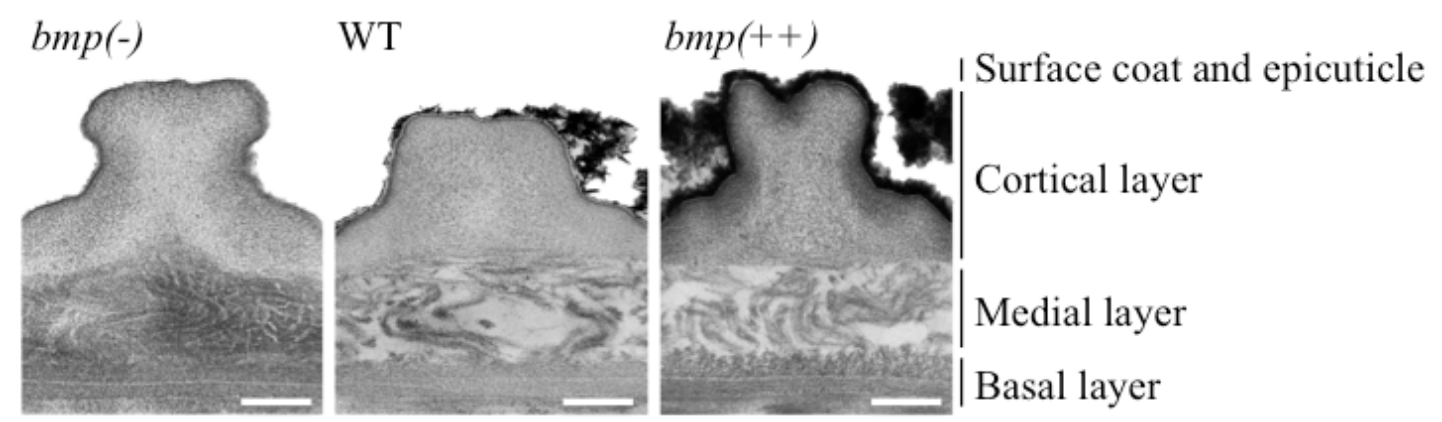

Fig. 3. The cuticle of animals with decreased (left), wild-type (middle), or increased (right) levels of BMP signaling. Scale bars $=0.2 \mu \mathrm{m}$. 